\title{
Pressure-induced collapse of large-moment magnetic order and localized-to-itinerant electronic transition in the host-guest compound $\left[\mathrm{Cs}_{6} \mathrm{Cl}\right]\left[\mathrm{Fe}_{24} \mathrm{Se}_{26}\right]$
}

\author{
Peter Adler $\odot,{ }^{1, *}$ Sergey A. Medvedev $\odot,{ }^{1}$ Martin Valldor, ${ }^{1,2}$ Pavel G. Naumov, ${ }^{1,3}$ \\ Moaz A. ElGhazali, ${ }^{1}$ and Rudolf Rüffer ${ }^{4}$ \\ ${ }^{1}$ Max Planck Institute for Chemical Physics of Solids, D-01187 Dresden, Germany \\ ${ }^{2}$ Centre for Materials Science and Nanotechnology (SMN), Department of Chemistry, University of Oslo, \\ P.O. Box 1033 Blindern, N-0315 Oslo, Norway \\ ${ }^{3}$ Shubnikov Institute of Crystallography of Federal Scientific Research Centre "Crystallography and Photonics" of Russian \\ Academy of Sciences, Moscow 119333, Russia \\ ${ }^{4}$ European Synchrotron Radiation Facility, CS 40220, F-38043 Grenoble Cedex 9, France
}

(Received 13 December 2019; revised manuscript received 9 March 2020; accepted 11 March 2020; published 31 March 2020)

\begin{abstract}
The magnetic properties of iron chalcogenides and pnictides have found much interest as magnetic fluctuations are suggested to drive the formation of Cooper pairs in iron-based superconductors. Here, we have studied the pressure dependence of the magnetic and electrical properties of the iron-selenide compound $\left[\mathrm{Cs}_{6} \mathrm{Cl}\right]\left[\mathrm{Fe}_{24} \mathrm{Se}_{26}\right]$ by energy-domain synchrotron Mössbauer spectroscopy up to $17 \mathrm{GPa}$ and by resistivity measurements up to $45 \mathrm{GPa}$. Similar to the binary superconductor $\mathrm{Fe}_{1+x} \mathrm{Se}$, the host-guest-type crystal structure of $\left[\mathrm{Cs}_{6} \mathrm{Cl}\right]\left[\mathrm{Fe}_{24} \mathrm{Se}_{26}\right]$ contains edge-sharing $\mathrm{FeSe}_{4}$ units, but its ground state is antiferromagnetically ordered. A complex hyperfine pattern suggests a nontrivial spin structure like a spin spiral with large magnetic moments in the range 2 to $3 \mu_{\mathrm{B}}$ at ambient pressure. High pressure drastically suppresses the Néel temperature from $220 \mathrm{~K}$ at ambient pressure to below $30 \mathrm{~K}$ at $12 \mathrm{GPa}$. Between 5 and $10 \mathrm{GPa}$ the complex magnetic hyperfine pattern collapses and a low-moment magnetic ground state emerges at higher pressures. A concomitant decrease of the resistivity by several orders of magnitude indicates that the electronic system becomes more itinerant. Full metallization occurs near $25 \mathrm{GPa}$, but no sign of superconductivity down to $1.5 \mathrm{~K}$ was found at any pressure. It is suggested that the insulating ground state of $\left[\mathrm{Cs}_{6} \mathrm{Cl}\right]\left[\mathrm{Fe}_{24} \mathrm{Se}_{26}\right]$ corresponds to an orbitally selective Mott phase, which due to an increased bandwidth, is transformed into a Hund's metal state under pressure.
\end{abstract}

DOI: 10.1103/PhysRevB.101.094433

\section{INTRODUCTION}

The efforts to understand the origin of superconductivity in iron-based pnictides and chalcogenides [1] has led to a large number of experimental and theoretical studies aiming to unravel their basic electronic and magnetic properties [2-5]. The electronic properties of this class of compounds are often described by a multiorbital electron system featuring moderately strong Mott-Hubbard-type Coulomb repulsion $U$, sizable electronic bandwidth $W$, as well as pronounced Hund coupling $J_{\mathrm{H}}$. Depending on the relative strength of these interactions, either Mott-type localized systems ("Mottness") or strongly correlated Hund metal systems ("Hundness") may emerge, where in the latter case strong electron correlations are rather driven by $J_{\mathrm{H}}$ than by $U$. The degree of localization

\footnotetext{
*adler@cpfs.mpg.de
}

Published by the American Physical Society under the terms of the Creative Commons Attribution 4.0 International license. Further distribution of this work must maintain attribution to the author(s) and the published article's title, journal citation, and DOI. Open access publication funded by the Max Planck Society. may vary between orbitals in the multiorbital system leading to the so-called orbitally selective Mott phase (OSMP) [6]. This nontrivial electronic situation may explain seemingly conflicting experimental observations, including frequent coexistence of rather localized electrons having large magnetic moments with itinerant electrons with low magnetic moments.

The crystal structures of most of the superconducting iron pnictides and chalcogenides are build up from layers of edgesharing $\mathrm{Fe} X_{4}$ tetrahedral units, forming a square lattice of $\mathrm{Fe}$ atoms. Starting from a nonsuperconducting magnetic parent compound, superconductivity can be induced either by doping or by applying external pressure. A special case is the binary $\mathrm{Fe}_{1+x} \mathrm{Se}$ system [7], which is not directly related to a magnetic parent compound, but its close vicinity to a magnetic state is apparent from muon spin rotation studies where pressureinduced static magnetic order with low magnetic moments appears above $\sim 0.8 \mathrm{GPa}$ [8]. Magnetic order and superconductivity coexist on the microscale in this pressure regime. A remarkable addition to the family of iron-based superconductors is $\mathrm{BaFe}_{2} \mathrm{~S}_{3}$, where the $\mathrm{FeS}_{4}$ units form a two-leg spin ladder. At ambient pressure $\mathrm{BaFe}_{2} \mathrm{~S}_{3}$ is a magnetic insulator with a stripe-type spin structure but it undergoes a pressure-driven metallization near $10 \mathrm{GPa}$ concurring with the appearance of superconductivity [9]. Transition temperatures $T_{\mathrm{c}}$ of up to 
$24 \mathrm{~K}$ were observed [10]. A benefit of iron-based superconductors and their relatives is that their high-pressure magnetic and structural properties can be conveniently studied by ${ }^{57} \mathrm{Fe}$ Mössbauer spectroscopy [11-15]. Using high-pressure synchrotron Mössbauer spectroscopy in the time domain (nuclear forward scattering) the magnetic phase diagram of $\mathrm{BaFe}_{2} \mathrm{~S}_{3}$ for pressures up to $10 \mathrm{GPa}$ was established [16]. Below $10 \mathrm{GPa}$ the antiferromagnetic ordering is retained, whereas near $10 \mathrm{GPa}$, i.e., in the pressure range of the insulator-metal transition, the magnetic order is suppressed. The electronic and magnetic behavior of $\mathrm{BaFe}_{2} \mathrm{~S}_{3}$ was interpreted in terms of a bandwidth-driven Mott insulator-metal transition $[9,10,16]$, where high pressure strengthens the Fe $d$-Se $p$ hybridization. Accordingly, the electronic bandwidth $W$ is enhanced and thus the $U / W$ ratio is reduced. This scenario was challenged [17], and it was argued that $\mathrm{BaFe}_{2} \mathrm{~S}_{3}$ is not a Mott-type insulator at ambient pressure, but that its insulating state emerges from a Hund metal state where strong orbital-selective electron correlations occur due to strong Hund exchange coupling $J_{\mathrm{H}}$. The insulating state then is a weakly coherent state with high effective masses for electrons in the vicinity of the Fermi level. The electron correlations are reduced under pressure, which leads to metallization. Also the selenium analog $\mathrm{BaFe}_{2} \mathrm{Se}_{3}$ shows a pressure-driven metallization with emerging superconductivity ( $T_{\mathrm{c}}$ up to $11 \mathrm{~K}$ at $\sim 13 \mathrm{GPa}$ ) [18], but the ambient pressure magnetic state differs from that of $\mathrm{BaFe}_{2} \mathrm{~S}_{3}$ in that it forms blocks containing clusters of four ferromagnetically coupled $\mathrm{Fe}$ ions that are antiferromagnetically aligned [19]. The magnetic state of $\mathrm{BaFe}_{2} \mathrm{Se}_{3}$ is possibly related to orbital ordering [20] and the ordered magnetic moments of the $\mathrm{Fe}$ atoms at ambient pressure are considerably higher $\left(\sim 2.8 \mu_{\mathrm{B}}\right)$ than those of $\mathrm{BaFe}_{2} \mathrm{~S}_{3}\left(\sim 1.2 \mu_{\mathrm{B}}\right)$. By contrast to $\mathrm{BaFe}_{2} \mathrm{~S}_{3}$, the pressure-induced superconducting state in $\mathrm{BaFe}_{2} \mathrm{Se}_{3}$ seems to appear in a pressure range where magnetic ordering is not yet suppressed [18,21].

The remarkable properties of $\mathrm{BaFe}_{2} X_{3}(X=\mathrm{S}, \mathrm{Se})$ suggest that it may be worth to explore the high-pressure behavior of a broader variety of insulating iron chalcogenides in order to unravel in more detail the underlying physics that determines their electronic and magnetic properties and even to search for pressure-induced superconductivity in other systems. Recently, we have reported the synthesis, structural, and magnetic properties of a complex host-guest-type iron selenide $\left[\mathrm{Cs}_{6} \mathrm{Cl}\right]\left[\mathrm{Fe}_{24} \mathrm{Se}_{26}\right]$ [22]. In its crystal structure basic tetrahedral $\mathrm{FeSe}_{4}$ units constitute a three-dimensional (3D) network of fused $\left[\mathrm{Fe}_{8} \mathrm{Se}_{6} \mathrm{Se}_{8 / 3}\right]$ cubane-type clusters (Fig. 1). In this host lattice the $\left[\mathrm{Cs}_{6} \mathrm{Cl}\right]^{5+}$ ions are incorporated as guests. X-ray absorption as well as Mössbauer spectra revealed that iron is in the +2 oxidation state. The requirement of charge neutrality indicates a small amount of iron deficiency compared to the ideal stoichiometry, or alternatively, one electron hole could be delocalized over the cuban-type units. $\left[\mathrm{Cs}_{6} \mathrm{Cl}\right]\left[\mathrm{Fe}_{24} \mathrm{Se}_{26}\right]$ is an antiferromagnet with a Néel temperature $T_{\mathrm{N}}=221 \mathrm{~K}$. A complex hyperfine pattern in ${ }^{57} \mathrm{Fe}$ Mössbauer spectra of the magnetically ordered state [22] indicates the formation of a complex spin structure. Resistivity measurements revealed semiconducting behavior with a quite small activation energy for electron transport, which together with the Mössbauer parameters suggests that the electronic system may be rather itinerant and not too far

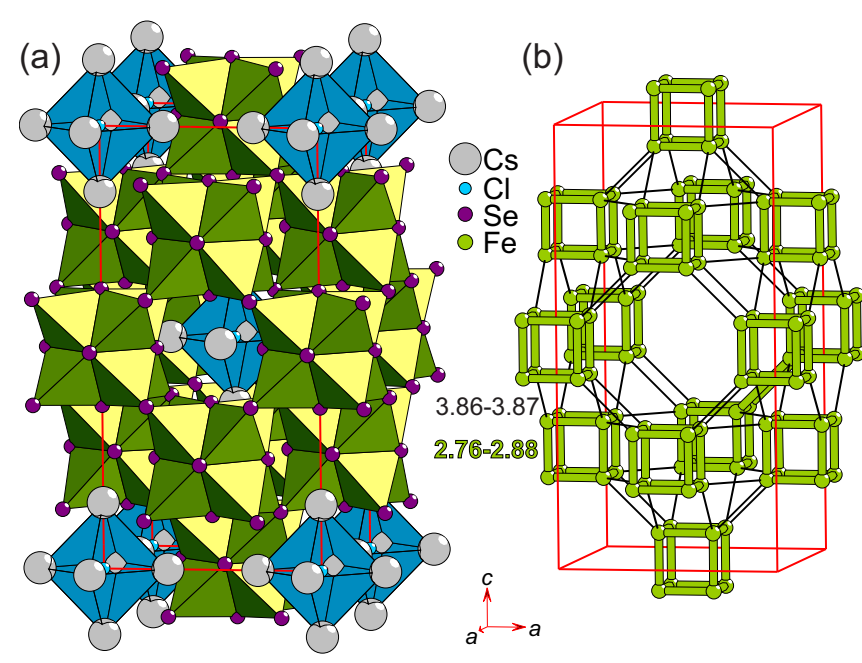

FIG. 1. Illustration of the crystal structure of $\left[\mathrm{Cs}_{6} \mathrm{Cl}\right]\left[\mathrm{Fe}_{24} \mathrm{Se}_{26}\right]$. (a) Perspective view of the crystal structure of the title compound. Polyhedra of $\mathrm{FeSe}_{4}$ and $\left[\mathrm{Cs}_{6} \mathrm{Cl}\right]$ are shown to emphasize the relative positions of different building units. (b) The Fe lattice with $\mathrm{Cs}$, $\mathrm{Cl}$, and Se removed. The two different interatomic distance ranges (in $\AA$ ) are shown: short distances with thick and long with thin lines, respectively.

from an insulator-metal transition. In the present work we explored the high-pressure magnetic and transport behavior of $\left[\mathrm{Cs}_{6} \mathrm{Cl}\right]\left[\mathrm{Fe}_{24} \mathrm{Se}_{26}\right]$ by using a combination of energy-domain synchrotron Mössbauer spectroscopy up to $17 \mathrm{GPa}$ and resistivity measurements up to $45 \mathrm{GPa}$. It will be shown that the complex large-moment magnetic order collapses between about 5 and $10 \mathrm{GPa}$. Above $10 \mathrm{GPa}$ a low-moment magnetic state seems to be formed. A drastic decrease in the electrical resistivity at high pressures suggests that the collapse of the large-moment magnetic order is due to enhanced electronic itinerancy at high pressure, but superconductivity does not appear down to $1.5 \mathrm{~K}$.

\section{EXPERIMENTAL DETAILS}

Inside an argon filled glove-box, $\left[\mathrm{Cs}_{6} \mathrm{Cl}\right]\left[\mathrm{Fe}_{24} \mathrm{Se}_{26}\right]$ enriched with $20 \%{ }^{57} \mathrm{Fe}$ was synthesized as follows: in-house made $\mathrm{Cs}_{2} \mathrm{Se}$ [22] was homogenized with powders of ultradry $\mathrm{CsCl}$ (3N TRC), Fe-metal (3N Alfa), ${ }^{57} \mathrm{Fe}$-metal (ISOFLEX), and elemental $\mathrm{Se}(5 \mathrm{~N}$ Alfa) in the relative stoichiometric amounts 2.5:1:19.2:4.8:23.5. The powder mixture was pressed into a pellet and placed in a corundum crucible inside a silica tube. The sample was thrice heated at $500^{\circ} \mathrm{C}$ in a water-cooled resistance furnace inside the glove-box, with intermediate grindings and pelletizations, for a total reaction time of $100 \mathrm{~h}$. Heating and cooling rates for each reaction were kept at $100^{\circ} \mathrm{Ch}^{-1}$. The ${ }^{57} \mathrm{Fe}$-enriched sample is denoted as $\left[\mathrm{Cs}_{6} \mathrm{Cl}\right]\left[{ }^{57} \mathrm{Fe}_{24} \mathrm{Se}_{26}\right]$.

Mössbauer spectra at ambient pressure were collected with a conventional WISSEL Mössbauer spectrometer that was operated in constant acceleration mode and used a ${ }^{57} \mathrm{Co} / \mathrm{Rh}$ radioactive source. A few $\mathrm{mg}$ of $\left[\mathrm{Cs}_{6} \mathrm{Cl}\right]\left[{ }^{57} \mathrm{Fe}_{24} \mathrm{Se}_{26}\right]$ was diluted with boron nitride in order to ensure homogeneous distribution in a plexiglass sample container that was placed 
inside a JANIS SHI-850-5 closed cycle refrigerator. The spectra were evaluated with the program MossWinn [23].

Energy-domain Mössbauer spectra at pressures up to $17 \mathrm{GPa}$ were conducted between 3 and $293 \mathrm{~K}$ using the synchrotron Mössbauer source (SMS) at the nuclear resonance beamline ID18 of the European Synchrotron Radiation Facility (ESRF, Grenoble), generating $14.4-\mathrm{keV}$ radiation [24]. For the high pressure studies, diamond anvil cells (DACs) manufactured from the nonmagnetic alloy MP35N and equipped with Boehler-Almax design diamond anvils with $500-\mu \mathrm{m}$ culets were used. The powdered sample was loaded into a sample chamber of $200-\mu \mathrm{m}$ diameter that was obtained by preindenting a cavity of $40-\mu \mathrm{m}$ thickness into the tungsten gasket. Silicone oil was used as pressure-transmitting medium. The high-pressure cell was prepared inside a glove box within an argon atmosphere. The pressure was determined by the ruby luminescence method. The DACs were placed into a cryomagnetic system from CryoIndustries. The beam size of the SMS radiation was $9 \mu$ (vertical) and $16 \mu$ (horizontal), respectively. The velocity scale was calibrated with a $25 \mu$ $\alpha$-iron foil and the linewidth and center shift of the SMS were determined by measuring repeatedly the spectra of the single line absorber $\mathrm{K}_{2} \mathrm{Mg}^{57} \mathrm{Fe}(\mathrm{CN})_{6}$ having an intrinsic linewidth of $0.21 \mathrm{~mm} / \mathrm{s}$. The data analysis was performed by using the transmission integral and a version of the program MOSSWINN where a squared Lorentzian for describing the line shape of the SMS was implemented. The source linewidth thus obtained was about $3 \Gamma_{0}\left(\Gamma_{0}\right.$ is the natural linewidth of the $14.4 \mathrm{keV}$ transition of ${ }^{57} \mathrm{Fe}$ ). Most Mössbauer spectra were recorded without external magnetic field, but at $5 \mathrm{GPa}$ two spectra above and below the magnetic ordering temperature were obtained in a longitudinal magnetic field of $6 \mathrm{~T}$. All isomer shifts in this work are given relative to that of standard $\alpha$-iron.

The electrical resistance between 1.5 and $300 \mathrm{~K}$ and up to $45 \mathrm{GPa}$ was measured by the dc current van der Pauw technique in heating mode. For insulating against the metallic gasket, a mixture of cubic BN with epoxy was used. The electrical leads were cut from a 5- $\mu \mathrm{m}$-thick Pt foil. The sample chamber had a diameter of $150 \mu \mathrm{m}$ and an initial thickness of $40 \mu \mathrm{m}$. The powder sample was loaded without a pressure transmitting medium. The pressure was determined by the ruby luminescence method. The resistivity results shown in this work were obtained on a sample of $\left[\mathrm{Cs}_{6} \mathrm{Cl}\right]\left[\mathrm{Fe}_{24} \mathrm{Se}_{26}\right]$ without ${ }^{57} \mathrm{Fe}$ enrichment from our previous study [22] due to the higher sample purity. However, for checking the reproducibility, high-pressure resistivity measurements on the ${ }^{57} \mathrm{Fe}$ enriched sample were also performed.

\section{RESULTS}

\section{A. Ambient pressure characterization}

According to powder x-ray diffraction (XRD) data the title compound is about 95 vol. \% pure, having $1 \% \mathrm{CsCl}$, $2 \% \mathrm{Fe}_{3} \mathrm{Se}_{4}$ [25], and 1-2\% of an unknown secondary phase. The main compound is the title compound, as diffraction intensities match very well with expectations and the refined unit cell parameters $(a=11.074(3)$ and $c=22.10(1) \AA$ only deviate less than $\sim 0.2 \%$ as compared to the previously re-

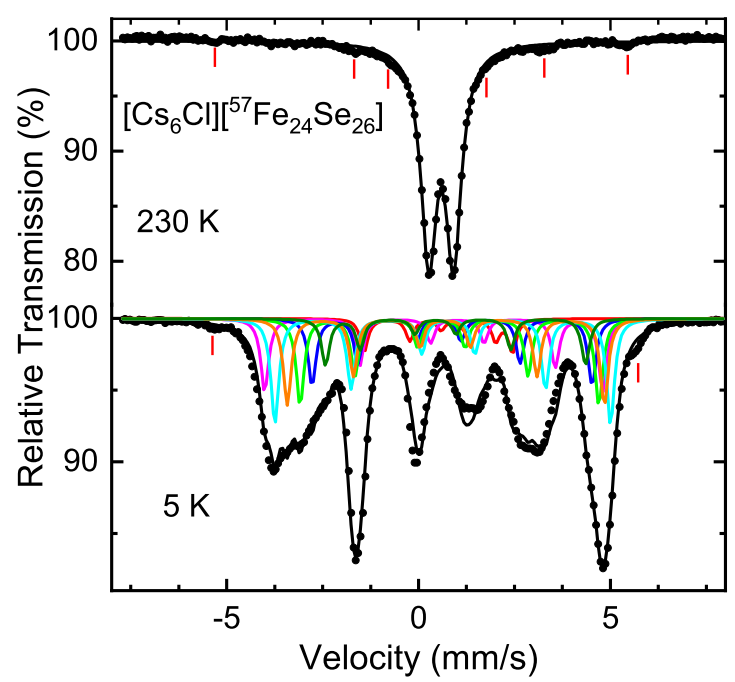

FIG. 2. Laboratory Mössbauer spectra of $\left[\mathrm{Cs}_{6} \mathrm{Cl}\right]\left[{ }^{57} \mathrm{Fe}_{24} \mathrm{Se}_{26}\right]$ at ambient pressure and the indicated temperatures. Solid black lines correspond to the total calculated spectrum, colored lines indicate decomposition into subspectra in order to mimic the complex hyperfine pattern. Vertical bars indicate lines due to a magnetic impurity phase.

ported ones ( $a=11.0991(9)$ and $c=22.143(2) \AA$ [22]) from the nonenriched compound.

In Fig. 2 laboratory Mössbauer spectra of $\left[\mathrm{Cs}_{6} \mathrm{Cl}\right]$ $\left[{ }^{57} \mathrm{Fe}_{24} \mathrm{Se}_{26}\right]$ at ambient pressure are shown. In agreement with our previous study [22], the spectrum at $230 \mathrm{~K}$ can be described by a single quadrupole doublet, which arises from the two crystallographically distinct sites that are, however, similar in their local coordination environment and thus not resolved in the spectrum. The spectrum confirms that the material is magnetically disordered at $230 \mathrm{~K}$. In contrast to the earlier sample, a weak additional hyperfine pattern is discernible, which is assigned to an impurity. The isomer shift of the doublet, IS $=0.58 \mathrm{~mm} / \mathrm{s}$ at $230 \mathrm{~K}$, is in good agreement with the earlier data, whereas quadrupole splitting $(\mathrm{QS})=$ $0.64 \mathrm{~mm} / \mathrm{s}$ is slightly larger than for the previous sample (QS $=0.58 \mathrm{~mm} / \mathrm{s}$ ). This may indicate a slightly different local environment in the two samples of the host-guest-type material. On the other hand, as in Ref. [22], a complicated hyperfine pattern is found at $5 \mathrm{~K}$, the shape of which was approximated by assuming a superposition of seven sextets differing in their hyperfine fields $B_{\mathrm{hf}}$ and quadrupole splitting parameters QS', but having a single $I S=0.70 \mathrm{~mm} / \mathrm{s}$. Here, QS' is determined by the quadrupole coupling constant $e Q V_{\mathrm{ZZ}}$ as well as by the orientation of $B_{\mathrm{hf}}$ with respect to the electric field gradient. Q denotes the nuclear quadrupolar moment and $V_{\mathrm{ZZ}}$ is the main component of the electric field gradient tensor in the principal axis system. The intensities and linewidths were allowed to vary. Due to the quite large absorber thickness arising from ${ }^{57} \mathrm{Fe}$ enrichment and the strongly overlapping lines, the spectra were evaluated using the transmission integral. The shape of the spectrum indicates a nontrivial spin arrangement. Complex hyperfine patterns were also found in the Mössbauer spectra of the magnetic phases of other iron chalcogenides, for instance for the block-type magnetic structure in $\mathrm{BaFe}_{2} \mathrm{Se}_{3}$ $[26,27]$ or for the incommensurate collinear spin-density wave 

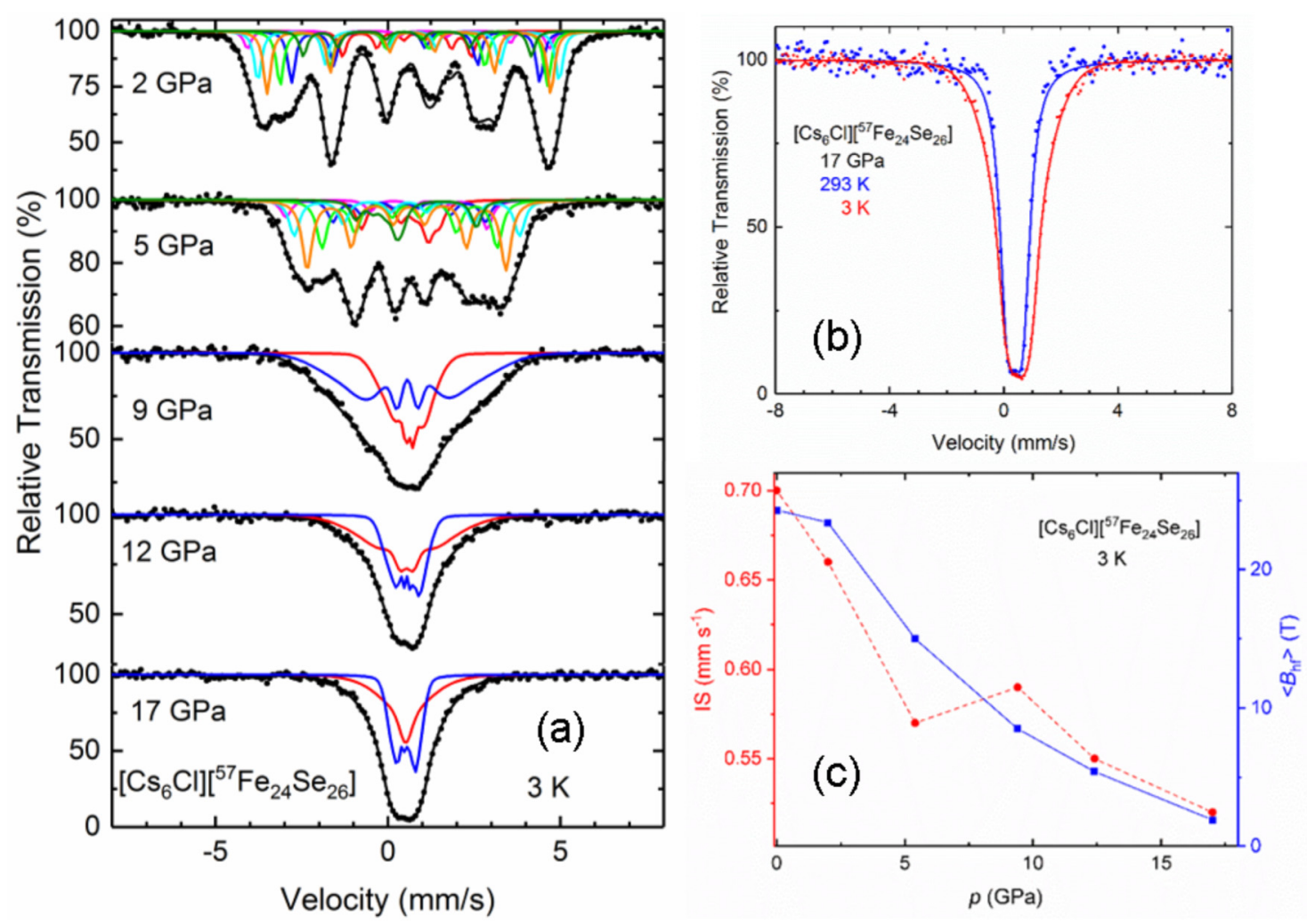

FIG. 3. (a) SMS spectra of $\left[\mathrm{Cs}_{6} \mathrm{Cl}\right]\left[{ }^{57} \mathrm{Fe}_{24} \mathrm{Se}_{26}\right]$ at base temperature $(\sim 3 \mathrm{~K})$ and the indicated pressures. Solid black lines correspond to

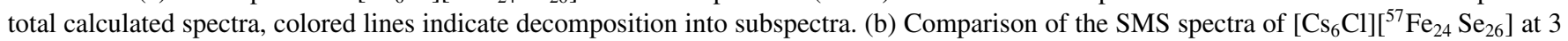
and $293 \mathrm{~K}$ obtained at $17 \mathrm{GPa}$. Line broadening at $3 \mathrm{~K}$ indicates the persistence of a small hyperfine field. (c) Pressure dependence of isomer shift (left scale, red) and average hyperfine field (right scale, blue) of $\left[\mathrm{Cs}{ }_{6} \mathrm{Cl}\right]\left[{ }^{57} \mathrm{Fe}_{24} \mathrm{Se}_{26}\right]$ at base temperature $(\sim 3 \mathrm{~K}$ ). Typically, the errors in IS are $\leqslant 0.01 \mathrm{~mm} / \mathrm{s}$ and in $\left\langle B_{\mathrm{hf}}\right\rangle$ about $0.1 \mathrm{~T}$ up to $5 \mathrm{GPa}$ and $0.4 \mathrm{~T}$ at higher pressures. Solid and dashed lines are guides to the eyes.

phase in $\mathrm{Fe}_{1.125} \mathrm{Te}$ [28]. The broad asymmetric shape of the low-temperature spectrum of $\left[\mathrm{Cs}_{6} \mathrm{Cl}\right]\left[\mathrm{Fe}_{24} \mathrm{Se}_{26}\right]$ suggests that a spiral spin structure may be formed (cf. the shape of the spectra of the spiral magnet $\mathrm{Sr}_{2} \mathrm{FeO}_{4}$ [29]), which leads to anisotropic contributions to the hyperfine field and to differing orientations between the hyperfine field and the electric field gradient due to the spin rotation of the magnetic moments within the spin spiral. This can explain the variation in the amplitude of the hyperfine fields as well as the different sizes and signs of the quadrupole splitting parameters QS' in our phenomenological fits. The actual distribution of $B_{\mathrm{hf}}$ and QS' values is probably even more complex and in the case of an incommensurate spin structure it is expected to be rather quasicontinuous [30]. The $B_{\mathrm{hf}}$ values are in the range between about 20 and $27 \mathrm{~T}$, which is comparable to $B_{\mathrm{hf}}$ values in $\mathrm{BaFe}_{2} \mathrm{Se}_{3}$ [27]. For the latter a magnetic moment of $2.8 \mu_{\mathrm{B}}$ was found from neutron diffraction studies [19]. Thus, a comparable size of the magnetic moment can be anticipated for $\left[\mathrm{Cs}_{6} \mathrm{Cl}\right]\left[\mathrm{Fe}_{24} \mathrm{Se}_{26}\right]$ at ambient pressure.

\section{B. High-pressure Mössbauer spectroscopy}

The evolution with pressure of the SMS spectra at base temperature $(\sim 3 \mathrm{~K})$ is shown in Fig. 3. A complex hyperfine pattern with some structure is still observed up to $5 \mathrm{GPa}$, but at higher pressures the structure collapses. At $17 \mathrm{GPa}$ only a quite symmetric signal is found, indicating that the magnetic order could be totally suppressed. However, comparison of the low-temperature spectrum with that at $293 \mathrm{~K}$ [Fig. 3(b)], where any magnetic ordering can be excluded, indicates that there is still a significant broadening corresponding to a small residual average $B_{\mathrm{hf}}$ of about $2 \mathrm{~T}$. Thus, a low-moment magnetic state still seems to be present at base temperature and $17 \mathrm{GPa}$. In any case, it is apparent that the average $B_{\mathrm{hf}}$ drastically decreases with pressure [Fig. 3(c)] above 5 GPa. The decrease of IS with increasing pressure corresponds to an increased $s$-electron density at the nucleus due to the volume decrease under pressure. It cannot be safely concluded from the limited number of pressure points whether there is an anomaly in IS $(p)$ near $5 \mathrm{GPa}$ or not. However, the magnitude of the pressure dependence of IS is of the expected order and there are no indications for a major pressure-induced change in electronic structure.

The temperature dependence of the Mössbauer spectra at different pressures is depicted in Figs. 4(a)-4(d). Due to the limited numbers of data points it is not possible to determine the detailed values of the magnetic ordering temperatures $T_{\mathrm{N}}$, nevertheless the trends are evident. While the spectra at $2 \mathrm{GPa}$ reveal hyperfine patterns up to about $200 \mathrm{~K}$, and thus $T_{\mathrm{N}}$ is expected to be only slightly reduced compared to ambient pressure $\left(T_{\mathrm{N}} \sim 221 \mathrm{~K}\right.$ from specific heat data [22]), there is a drastic decrease in $T_{\mathrm{N}}$ for higher pressures. Even at $5 \mathrm{GPa}$ $T_{\mathrm{N}}$ has decreased by more than 100 to about $80 \mathrm{~K}$. A minority hyperfine pattern, still visible in the spectrum at $5 \mathrm{GPa}$ and 

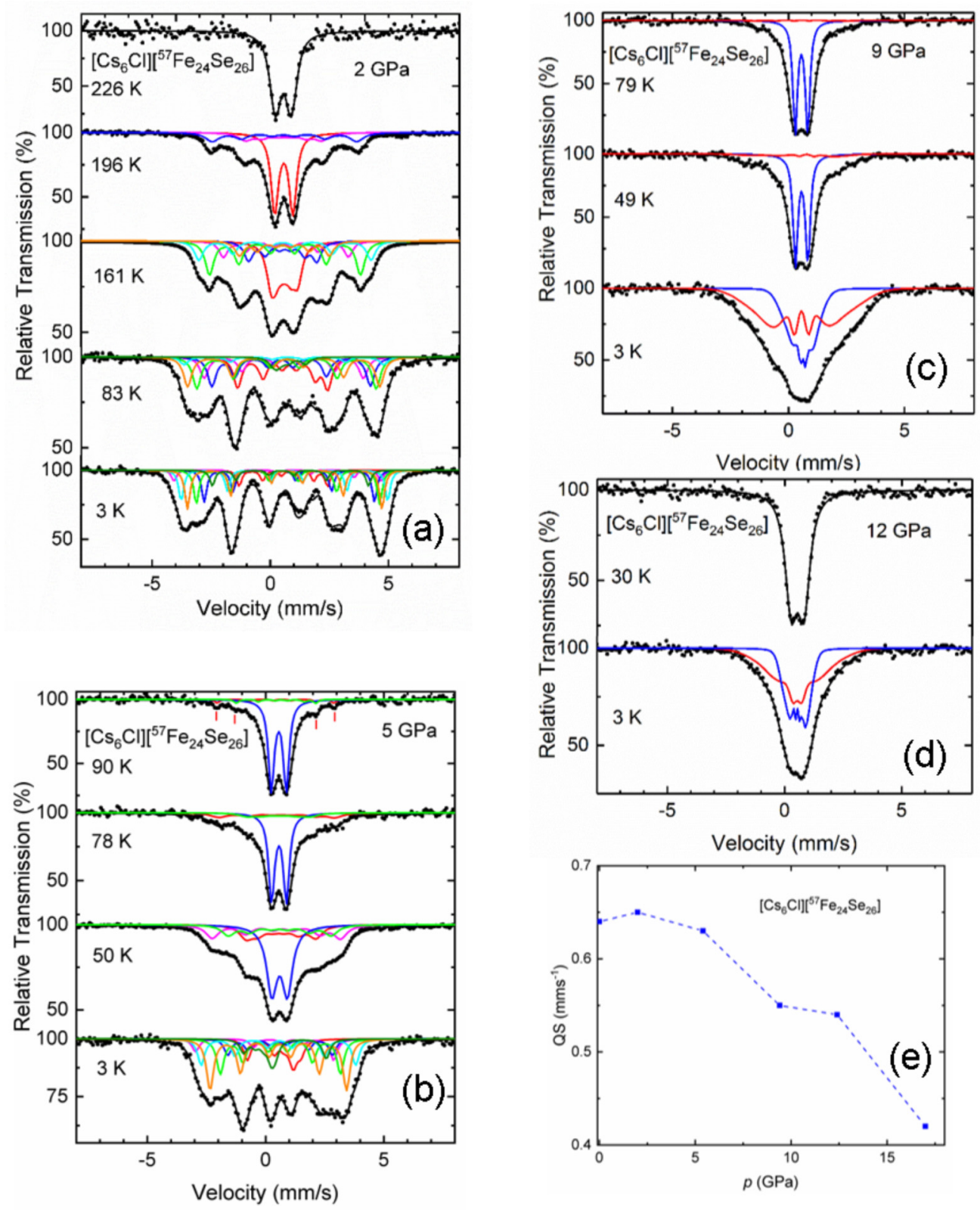

FIG. 4. (a)-(d): Temperature dependence of the SMS spectra of $\left[\mathrm{Cs}_{6} \mathrm{Cl}\right]\left[{ }^{57} \mathrm{Fe}_{24} \mathrm{Se}_{26}\right]$ at 2, 5, 9, and 12 GPa, respectively. Solid black lines correspond to total calculated spectra, colored lines indicate decomposition into subspectra. (e) Pressure dependence of the quadrupole splitting in the paramagnetic phase. The data points are for temperatures slightly above the Néel temperature, except for $17 \mathrm{GPa}$, where only the room temperature value was measured. The errors in QS are $\leqslant 0.01 \mathrm{~mm} / \mathrm{s}$. The dashed line is a guide to the eyes. The vertical bars in (b) indicate quite sharp lines at $90 \mathrm{~K}$, which most likely arise from a magnetic impurity phase.

$90 \mathrm{~K}$ with relatively sharp lines, may rather correspond to the impurity seen in the ambient pressure spectra. Further increase of pressure to 9 and $12 \mathrm{GPa}$ results in a further decrease of $T_{\mathrm{N}}$ to below $50 \mathrm{~K}$, and $30 \mathrm{~K}$, respectively. The strong reduction in $B_{\mathrm{hf}}$ with pressure is a signature for a corresponding reduction of the ordered magnetic moments and together with the strong reduction in $T_{\mathrm{N}}$ it suggests that the complex spin structure with quite large magnetic moments is suppressed above $5 \mathrm{GPa}$ and transformed into a magnetic state with low moments that persists up to at least $17 \mathrm{GPa}$. These results correspond to a rather itinerant electronic system, which is corroborated by the pressure-dependent resistivity data presented below. Finally, it needs to be considered whether the changes in the magnetic structure may be related to changes in the crystal structure under pressure as predicted for $\mathrm{BaFe}_{2} \mathrm{Se}_{3}$ [21] or experimentally found for $\mathrm{TlFe}_{1.6} \mathrm{Se}_{2}$ [14]. Within the present work no direct structural studies under pressure have been performed, but some information can be gained from the pressure dependence of the quadrupole splitting in the paramagnetic phase. In Fig. 4(e) the pressure dependence of the quadrupole splitting in the paramagnetic phase at a temperature just above the magnetic phase transition is depicted (except for $17 \mathrm{GPa}$ where only the $293 \mathrm{~K}$ value was measured). At ambient pressure only a small temperature dependence of QS was found [22]. The data indicate that QS essentially is independent of pressure for $p \leqslant 5 \mathrm{GPa}$, while 


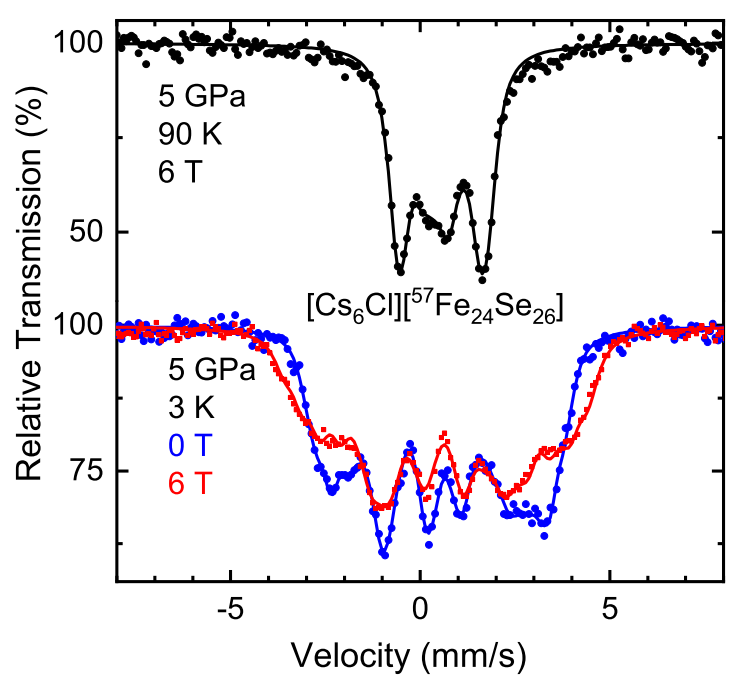

FIG. 5. Top: SMS spectrum of $\left[\mathrm{Cs}_{6} \mathrm{Cl}\right]\left[{ }^{57} \mathrm{Fe}_{24} \mathrm{Se}_{26}\right]$ at $5 \mathrm{GPa}$ and $90 \mathrm{~K}$ in a longitudinal field of $6 \mathrm{~T}$. The solid black line corresponds to the fit of the spectrum with a model assuming a randomly oriented electric field gradient in an external magnetic field. Bottom: Comparison of the SMS spectra at $5 \mathrm{GPa}$ and $3 \mathrm{~K}$ in zero field (blue) and in an applied longitudinal field of $6 \mathrm{~T}$ (red).

there is a significant but not drastic decrease between 5 and $9 \mathrm{GPa}$, i.e., just in the pressure range where the complex magnetic structure collapses. The pressure dependence of QS indicates that subtle structural modifications may occur in the pressure range of the magnetic structure collapse, but it may also reflect the increasing itinerancy of the electronic system in this pressure range. At $17 \mathrm{GPa}$ there seems to be a further reduction in QS, but here no data at low temperatures were measured. XRD studies under pressure are required to clarify structural details.

An advantage of high-pressure SMS experiments compared to laboratory high-pressure Mössbauer spectroscopy is that spectra at low temperatures and high pressures can be conveniently recorded in an external magnetic field. Mössbauer spectra at $5 \mathrm{GPa}$ were measured in an external longitudinal field of $6 \mathrm{~T}$ at $90 \mathrm{~K}$, i.e., above $T_{\mathrm{N}}$, and at $3 \mathrm{~K}$, i.e. below $T_{\mathrm{N}}$ (Fig. 5). The shape of the spectrum at $90 \mathrm{~K}$ can be well described using the model of a randomly oriented axially symmetric electric field gradient in an external magnetic field as implemented in the MOSSWINN program [23]. The fit parameters IS $=0.57(1) \mathrm{mm} / \mathrm{s}$ and $\mathrm{QS}=0.59(2) \mathrm{mm} / \mathrm{s}$ compare reasonably well with IS $=0.55(1) \mathrm{mm} / \mathrm{s}$ and $\mathrm{QS}=$ $0.63(1) \mathrm{mm} / \mathrm{s}$ obtained from the zero-field spectra. The fitted magnetic field $B=6.04(5) \mathrm{T}$ is identical to the external field, thus verifying the absence of any additional internal fields. On the other hand, the shape of the $3 \mathrm{~K}$ spectrum measured at $5 \mathrm{GPa}$ in zero-field differs considerably from that measured at $B=6 \mathrm{~T}$. Qualitatively it is seen that at $6 \mathrm{~T}$ components with increased $B_{\mathrm{hf}}$ values occur, whereas there is also some shift in $B_{\text {hf }}$ toward the center of the spectrum. These results are in agreement with a noncollinear antiferromagnetic spin structure like a spin spiral where a certain splitting of lines can be expected as the external field partly enhances and partly diminishes the internal hyperfine field.

\section{High-pressure resistivity studies}

The temperature dependence of the resistivity of the sample without ${ }^{57} \mathrm{Fe}$ enrichment measured in the DAC at low pressures (Fig. 6) is in good agreement with the previous ambient pressure data [22]: the thermally activated conductivity at high temperatures is followed by an additional resistivity increase due to antiferromagnetic spin ordering as the temperature decreases below the Néel temperature $T_{\mathrm{N}}$. The latter was derived from the inflection point in the plot of the resistivity versus inverse temperature [Fig. 6(a)]. The drastic decrease in $T_{\mathrm{N}}$ with increasing pressure from $192 \mathrm{~K}$ at $1.8 \mathrm{GPa}$ to $82 \mathrm{~K}$ at $5.5 \mathrm{GPa}$ (Fig. 7) compares well with the results from the Mössbauer studies (Fig. 4). At pressures above $7 \mathrm{GPa}$, no anomalies due to magnetic ordering could be observed in the temperature dependence of the resistivity, which is in agreement with the transition to a low-moment magnetic order as suggested by Mössbauer spectroscopy.

It is evident from Fig. 6(b) that the resistivity decreases continuously with increasing pressure by several orders of magnitude. High conductivity $\left(\sim 10^{3} \Omega^{-1} \mathrm{~cm}^{-1}\right)$ at low temperature and $\sim 10 \mathrm{GPa}$ suggests that the electronic system has already a rather itinerant character, but the temperature dependence of resistivity indicates that the sample still remains semiconducting up to pressures beyond $20 \mathrm{GPa}$. Above $25 \mathrm{GPa}$ the resistivity starts to increase with increasing temperature [inset in Fig. 6(b)] indicating the formation of a metallic state in $\left[\mathrm{Cs}_{6} \mathrm{Cl}\right]\left[\mathrm{Fe}_{24} \mathrm{Se}_{26}\right]$. No resistance drop indicative for superconductivity has been observed down to $1.5 \mathrm{~K}$ at any pressure up to $45 \mathrm{GPa}$. Due to higher sample purity we have presented here the resistivity data of the sample without ${ }^{57} \mathrm{Fe}$ enrichment, but similar results were also obtained for the ${ }^{57} \mathrm{Fe}$ enriched sample.

\section{DISCUSSION}

In the present work we have shown that the host-guesttype iron selenide compound $\left[\mathrm{Cs}_{6} \mathrm{Cl}\right]\left[\mathrm{Fe}_{24} \mathrm{Se}_{26}\right]$ undergoes a pressure-driven transition from a more localized to an itinerant electronic system with concomitant collapse of the possibly noncollinear large-moment magnetic order. Similar to superconducting iron chalcogenides and their magnetic relatives, the crystal structure of $\left[\mathrm{Cs}_{6} \mathrm{Cl}\right]\left[\mathrm{Fe}_{24} \mathrm{Se}_{26}\right]$ is composed of edge-sharing $\mathrm{FeSe}_{4}$ units, but here the Fe-Se structure is $3 \mathrm{D}$. The results from our combined high-pressure synchrotron Mössbauer and resistivity studies are summarized in the phase diagram of Fig. 7. Nonhydrostatic pressure conditions may alter details of the phase diagram, but the Mössbauer and resistivity results are consistent. The following general trends are evident: In the low-pressure range, up to about $5 \mathrm{GPa}$, $\left[\mathrm{Cs}_{6} \mathrm{Cl}\right]\left[\mathrm{Fe}_{24} \mathrm{Se}_{26}\right]$ features an insulating ground state and a complex spin structure. The shape of the Mössbauer spectra indicates that the magnetic state may correspond to a spiral spin arrangement. The magnetic hyperfine fields are in the range 20-27 $\mathrm{T}$, similar to other iron chalcogenides with insulating ground states $[14,27]$ and quite large magnetic moments $>2 \mu_{\mathrm{B}}$. The resistivity data show that with increasing pressure the electronic system becomes more itinerant, which is the origin for the collapse of the complex, large-moment magnetic state in the pressure range between about 5 and 

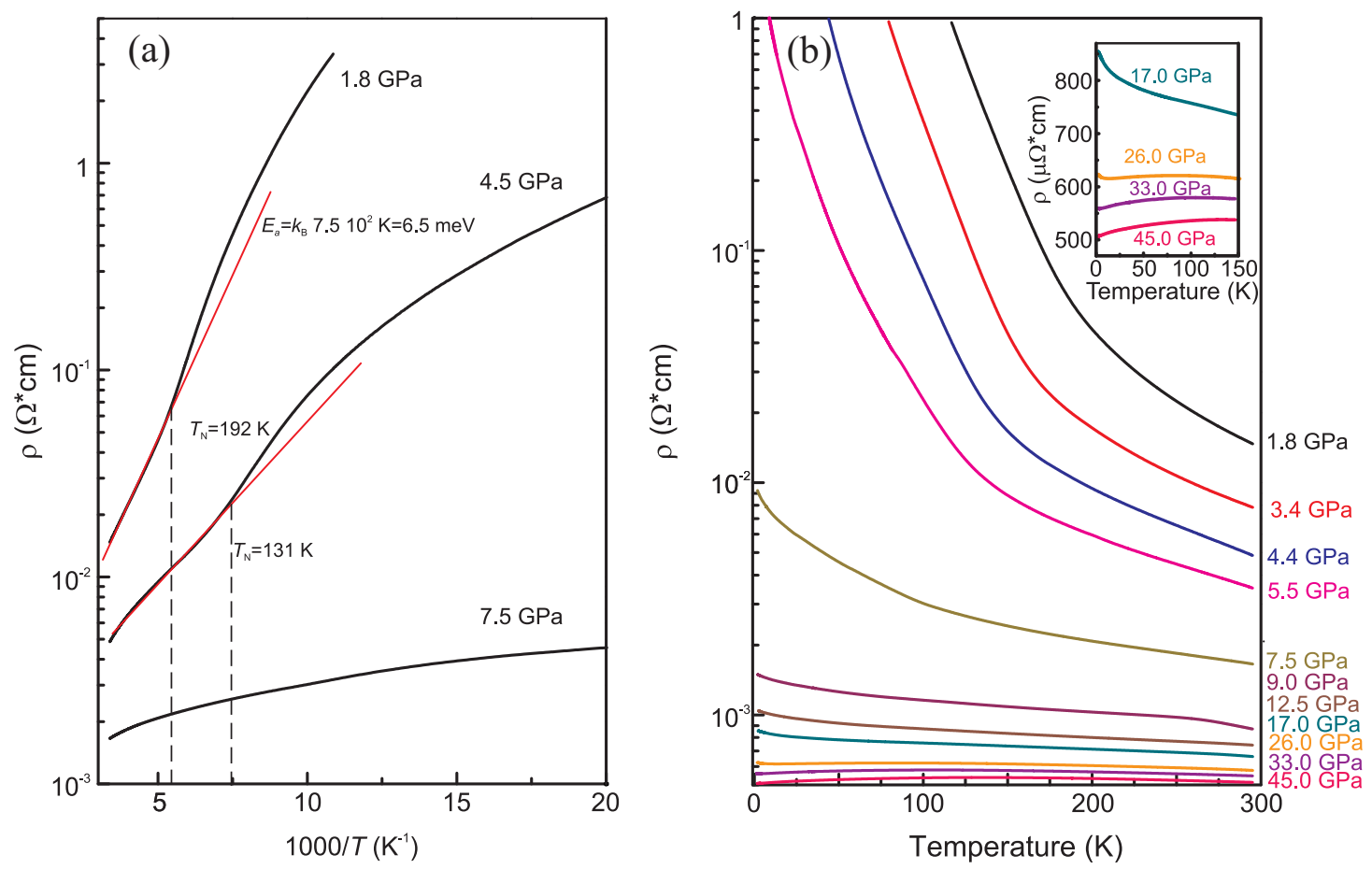

FIG. 6. Pressure effect on electronic transport properties of $\left[\mathrm{Cs}_{6} \mathrm{Cl}\right]\left[\mathrm{Fe}_{24} \mathrm{Se}_{26}\right]$. (a) Electrical resistivity as a function of inverse temperature at different pressures. Arrhenius fit to the high-temperature data (red solid line) has been used to estimate the activation energy. The Néel temperature is indicated by a vertical broken line. At pressures above 7 GPa no peculiarities due to AFM ordering were observed. (b) Pressure evolution of the temperature dependence of the electrical resistivity. Inset: The change in the slope of the temperature dependence of the resistivity from negative to positive indicates metallization at pressures above $26 \mathrm{GPa}$.

$10 \mathrm{GPa}$. The Mössbauer spectra suggest that a low-moment magnetic state is present at even higher pressures, up to at least $17 \mathrm{GPa}$ (highest pressure in this Mössbauer study). Up to about $25 \mathrm{GPa}$, the resistivity data show a semiconduct-

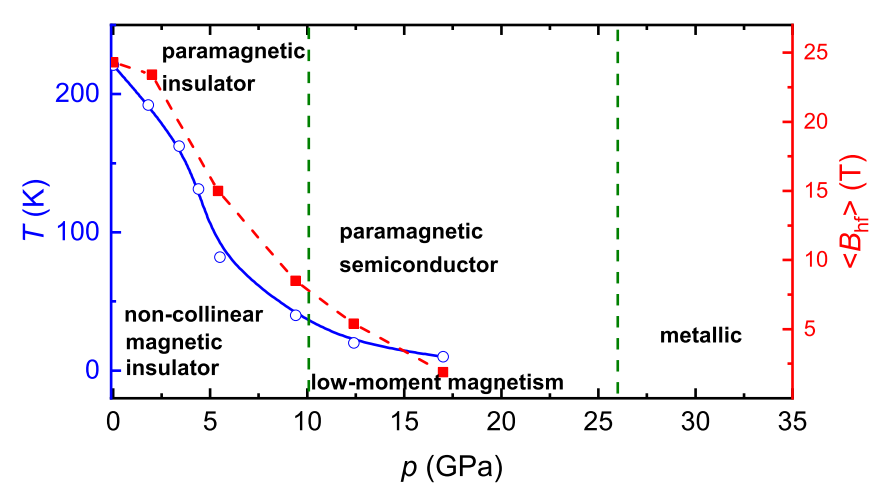

FIG. 7. Schematic phase diagram which summarizes the results of the high-pressure Mössbauer and resistivity studies for $\left[\mathrm{Cs}_{6} \mathrm{Cl}\right]\left[\mathrm{Fe}_{24} \mathrm{Se}_{26}\right]$. The blue circles correspond to the magnetic ordering temperatures (left scale), which up to $5.5 \mathrm{GPa}$ were determined from the resistivity data (error about $2 \mathrm{~K}$ ) and for higher pressures they correspond to upper limits roughly estimated from the Mössbauer data. The red squares show the average hyperfine fields obtained from the Mössbauer spectra, which reflect the size of the magnetic moments. Solid and dashed lines are guides to the eye. The vertical dashed lines tentatively separate the regions of the phase diagram. However, the crossover between the different regimes is rather gradual. ing temperature characteristics, whereas for higher pressures full metallization is reached. It can be anticipated that in this pressure range the magnetic moments are completely quenched.

No indications of superconductivity down to $1.5 \mathrm{~K}$ and for pressures up to $45 \mathrm{GPa}$ were found for $\left[\mathrm{Cs}_{6} \mathrm{Cl}\right]\left[\mathrm{Fe}_{24} \mathrm{Se}_{26}\right]$, but our high-pressure studies suggest that the basic electronic properties of the title system are comparable to those of other iron chalcogenides [31]. In particular, the phase diagram of Fig. 7 has remarkable similarities with the pressure-dependent phase diagram of the spin-ladder compound $\mathrm{BaFe}_{2} \mathrm{Se}_{3}[18,21]$, which at ambient pressure is an insulator with a block-type, large magnetic moment ground state and which reveals a pressure-driven reduction of the magnetic moment too. For $\mathrm{BaFe}_{2} \mathrm{Se}_{3}$ the transformation of the blocktype magnetic phase into a stripe-type magnetic order (CX structure) with smaller moments was suggested to occur near $12 \mathrm{GPa}$. In this pressure range $\mathrm{BaFe}_{2} \mathrm{Se}_{3}$ is already metallic. At even higher pressures, near about $30 \mathrm{GPa}$, the total loss of the magnetic moment was predicted [21]. Superconductivity in $\mathrm{BaFe}_{2} \mathrm{Se}_{3}$ was suggested to be related to the coexistence of the block-type and the CX-type spin structure, whereas in $\mathrm{BaFe}_{2} \mathrm{~S}_{3}$ it occurs in a zero-moment metallic state after total collapse of the magnetic order [10,16,21]. These results demonstrate the close relationship between superconductivity and magnetism in these systems, which may also rationalize the absence of superconductivity in $\left[\mathrm{Cs}_{6} \mathrm{Cl}\right]\left[\mathrm{Fe}_{24} \mathrm{Se}_{26}\right]$. Although the overall electronic situation appears to be comparable to $\mathrm{BaFe}_{2} \mathrm{Se}_{3}$, the spin structures are different due to different exchange pathways in the different crystal structures. 
In $\left[\mathrm{Cs}_{6} \mathrm{Cl}\right]\left[\mathrm{Fe}_{24} \mathrm{Se}_{26}\right]$ the magnetic structure does not seem to induce spin fluctuations favoring the formation of Cooper pairs.

The electronic situation in the iron chalcogenides is of great interest, as in terms of a Hubbard-type electronic structure model they correspond to an intermediate strength of electron correlations where the on-site electron repulsion $U$ and the bandwidth $W$ are of comparable size $(U / W \sim 1)$ [2]. The iron pnictides are expected to be more itinerant $(U / W<1)$, whereas the much-studied cuprates are more localized $(U / W>1)$. In the intermediate coupling regime, Hund's rule coupling $J_{\mathrm{H}}$ plays an important role for the electronic properties of multiorbital systems and may lead to the formation of an orbitally selective Mott phase revealing coexistence of localized gapped and itinerant zero-gap electron bands [6]. In fact, the spin ladder compound $\mathrm{BaFe}_{2} \mathrm{Se}_{3}$ was considered to be an example for an OSMP phase [32-34]. Given the similarities in the high-pressure phase diagrams of $\mathrm{BaFe}_{2} \mathrm{Se}_{3}$ and $\left[\mathrm{Cs}_{6} \mathrm{Cl}\right]\left[\mathrm{Fe}_{24} \mathrm{Se}_{26}\right]$, the ground state in $\left[\mathrm{Cs}_{6} \mathrm{Cl}\right]\left[\mathrm{Fe}_{24} \mathrm{Se}_{26}\right]$ may also correspond to an orbitally selective Mott insulator state. Increasing pressure tunes the system from the more localized to itinerant electronic behavior by decreasing the $U / W$ ratio as the bandwidth increases with pressure due to shorter bond distances and increased Fe-3dSe- $4 p$ hybridization strength. The large-moment magnetism is the signature of the more localized regime and thus is lost with increasing pressure, which is supported by the close relation between the changes in the Mössbauer spectra and in the resistivities under pressure in $\left[\mathrm{Cs}_{6} \mathrm{Cl}\right]\left[\mathrm{Fe}_{24} \mathrm{Se}_{26}\right]$. The highpressure metallic state then corresponds to a Hund's metal. The rather continuous evolution of the electronic behavior with pressure differs from the high-pressure phase diagram of a prototypical Mott insulator like $\mathrm{NiS}_{2}$ [35] where the localized and itinerant electronic regimes are separated. In the localized regime the magnetic ordering temperature slightly increases with pressure, whereas in the metallic regime, similar as for $\left[\mathrm{Cs}_{6} \mathrm{Cl}\right]\left[\mathrm{Fe}_{24} \mathrm{Se}_{26}\right]$, it decreases with increasing pressure. In the crossover region well-defined insulator-metal transitions are found in $\mathrm{NiS}_{2}$.

\section{SUMMARY}

We have studied the pressure dependence of the magnetic and electrical properties of a host-guest-type iron selenide compound $\left[\mathrm{Cs}_{6} \mathrm{Cl}\right]\left[\mathrm{Fe}_{24} \mathrm{Se}_{26}\right]$, the crystal structure of which is composed from edge-sharing $\mathrm{FeSe}_{4}$ units. In contrast to binary $\mathrm{Fe}_{1+x} \mathrm{Se}$, no superconductivity was observed down to $1.5 \mathrm{~K}$ and for pressures up to $45 \mathrm{GPa}$, instead $\left[\mathrm{Cs}_{6} \mathrm{Cl}\right]\left[\mathrm{Fe}_{24} \mathrm{Se}_{26}\right]$ is magnetically ordered. High pressure suppresses the nontrivial large-moment, possibly spiral, magnetic ground state and above $10 \mathrm{GPa}$ a low-moment magnetic state emerges. Concomitantly the electronic system becomes more itinerant and finally metallizes near $25 \mathrm{GPa}$. Similar as in case of the spin-ladder compound $\mathrm{BaFe}_{2} \mathrm{Se}_{3}$ the ground state may correspond to an orbitally selective Mott phase, which is transformed into a Hund's metal state under pressure. Clearly, the determination of the magnetic structure at ambient pressure is required for a more detailed theoretical analysis. Preliminary neutron diffraction data indeed indicate a nontrivial propagation vector.

\section{ACKNOWLEDGMENT}

We gratefully acknowledge the ESRF for providing beamtime at the Nuclear Resonance Beamline ID18.
[1] Q. Si, R. Yu, and E. Abrahams, High temperature superconductivity in iron pnictides and chalcogenides, Nat. Rev. Mater. 1, 16017 (2016).

[2] P. Dai, J. Hu, and E. Dagotto, Magnetism and its microscopic origin in iron-based high-temperature superconductors, Nat. Phys. 8, 709 (2012).

[3] N. Mannella, The magnetic moment enigma in Fe-based high temperature superconductors, J. Phys.: Condens. Matter 26, 473202 (2014).

[4] P. Dai, Antiferromagnetic order and spin dynamics in ironbased superconductors, Rev. Mod. Phys. 87, 855 (2015).

[5] E. Bascones, B. Valenzuela, and M. J. Calderón, Magnetic interactions in iron superconductors: A review, C. R. Phys. 17, 36 (2016)

[6] A. Georges, L. de' Medici, and J. Mravlje, Strong correlations from Hund's coupling, Annu. Rev. Condens. Matter Phys. 4, 137 (2013).

[7] A. J. Coldea and M. D. Watson, The key ingredients of the electronic structure of FeSe, Annu. Rev. Condens. Matter Phys. 9, 125 (2018).

[8] M. Bendele, A. Amato, K. Conder, M. Elender, H. Keller, H.-H. Klauss, H. Letkens, E. Pomjakushina, A. Raselli, and R.
Khasanov, Pressure Induced Static Magnetic Order in Superconducting $\mathrm{FeSe}_{1-x}$, Phys. Rev. Lett. 104, 087003 (2010).

[9] H. Takahashi, A. Sugimoto, Y. Nambu, T. Yamauchi, Y. Hirata, T. Kawakami, M. Avdeev, K. Matsubayashi, F. Du, C. Kawashima, H. Soeda, S. Nakano, Y. Uwatoko, Y. Ueda, T. J. Sato, and K. Ohgushi, Pressure-induced superconductivity in the iron-based ladder material $\mathrm{BaFe}_{2} \mathrm{~S}_{3}$, Nat. Mater. 14, 1008 (2015).

[10] T. Yamauchi, Y. Hirata, Y. Ueda, and K. Ohgushi, PressureInduced Mott Transition Followed by a 24-K Superconducting Phase in $\mathrm{BaFe}_{2} \mathrm{~S}_{3}$, Phys. Rev. Lett. 115, 246402 (2015).

[11] S. Medvedev, T. M. Queen, I. A. Troyan, T. Palasyuk, M. I. Eremets, R. J. Cava, S. Naghavi, F. Casper, V. Ksenofontov, G. Wortmann, and C. Felser, Electronic and magnetic phase diagram of $\beta$ - $\mathrm{Fe}_{1.01}$ Se with superconductivity at $36.7 \mathrm{GPa}$ under pressure, Nat. Mater. 8, 630 (2009).

[12] T. Kawakami, T. Kamatani, H. Okada, H. Takahashi, S. Nasu, Y. Kamihara, M. Hirano, and H. Hosono, High-pressure ${ }^{57} \mathrm{Fe}$ Mössbauer spectroscopy of LaFeAsO, J. Phys. Soc. Jpn. 78, 123703 (2009).

[13] V. Ksenofontov, S. A. Medvedev, L. M. Schoop, G. Wortmann, T. Palasyuk, V. Tsurkan, J. Deisenhofer, A. Loidl, and C. Felser, 
Superconductivity and magnetism in $\mathrm{Rb}_{0.8} \mathrm{Fe}_{1.6} \mathrm{Se}_{2}$ under pressure, Phys. Rev. B 85, 214519 (2012).

[14] P. G. Naumov, K. Filsinger, S. I. Shylin, O. I. Barkalov, V. Ksenofontov, Y. Qi, T. Palasyuk, W. Schnelle, S. A. Medvedev, M. Greenblatt, and C. Felser, Pressure-induced magnetic collapse and metallization of $\mathrm{TlFe}_{1.6} \mathrm{Se}_{2}$, Phys. Rev. B 96, 064109 (2017).

[15] P. Materne, W. Bi, J. Zhao, M. Y. Hu, R. Kappenberger, S. Wurmehl, S. Aswartham, B. Büchner, and E. E. Alp, Microscopic phase diagram of $\mathrm{LaFeAsO}$ single crystals under pressure, Phys. Rev. B 98, 174510 (2018).

[16] P. Materne, W. Bi, J. Zhao, M. Y. Hu, M. L. Amigó, S. Seiro, S. Aswartham, B. Büchner, and E. E. Alp, Bandwith controlled insulator-metal transition in $\mathrm{BaFe}_{2} \mathrm{~S}_{3}$ : A Mössbauer study under pressure, Phys. Rev. B 99, 020505(R) (2019).

[17] J. M. Pizarro and E. Bascones, Strong electronic correlations and Fermi surface reconstruction in the quasi-one-dimensional iron superconductor $\mathrm{BaFe}_{2} \mathrm{~S}_{3}$, Phys. Rev. Mater. 3, 014801 (2019).

[18] J. Ying, H. Lei, C. Petrovic, Y. Xiao, and V. V. Struzhkin, Interplay of magnetism and superconductivity in the compressed Fe-ladder $\mathrm{BaFe}_{2} \mathrm{Se}_{3}$, Phys. Rev. B 95, 241109(R) (2017).

[19] Y. Nambu, K. Ohgushi, S. Suzuki, F. Du, M. Avdeev, Y. Uwatoko, K. Monakata, H. Fukazawa, S. Chi, Y. Ueda, and T. J. Sato, Block magnetism coupled with local distortion in the iron-based spin-ladder compound $\mathrm{BaFe}_{2} \mathrm{Se}_{3}$, Phys. Rev. B 85, 064413 (2012).

[20] T. Aoyama, S. Imaizumi, T. Togashi, Y. Sato, K. Hashizume, Y. Nambu, Y. Hirata, M. Matsubara, and K. Ohgushi, Polar state induced by block-type lattice distortions in $\mathrm{BaFe}_{2} \mathrm{Se}_{3}$ with quasi-one-dimensional ladder structure, Phys. Rev. B 99, 241109(R) (2019).

[21] Y. Zhang, L.-F. Lin, J.-L. Zhang, E. Dagotto, and S. Dong, Sequential structural and antiferromagnetic transitions in $\mathrm{BaFe}_{2} \mathrm{Se}_{3}$ under pressure, Phys. Rev. B 97, 045119 (2018).

[22] M. Valldor, B. Böhme, Y. Prots, H. Borrmann, P. Adler, W. Schnelle, Y. Watier, C. Y. Kuo, T.-W. Pi, Z. Hu, C. Felser, and L. H. Tjeng, $\left[\mathrm{Cs}_{6} \mathrm{Cl}\right]\left[\mathrm{Fe}_{24} \mathrm{Se}_{26}\right]$ : A host-guest compound with unique Fe-Se topology, Chem. Eur. J. 22, 4626 (2016).

[23] Z. Klencsár, E. Kuzmann, and A. Vértes, User-friendly software for Mössbauer spectrum analysis, J. Radioanal. Nucl. Chem. 210, 105 (1996).

[24] V. Potapkin, A. I. Chumakov, G. V. Smirnov, J.-P. Celse, R. Rüffer, C. McCammon, and L. Dubrovinski, The ${ }^{57} \mathrm{Fe}$ synchrotron Mössbauer source at the ESRF, J. Synchrotron Rad. 19, 559 (2012).

[25] A. Okazaki and K. Hirakawa, Structural study of iron selenides $\mathrm{FeSe}_{x}$. I ordered arrangement of defects of Fe atoms, J. Phys. Soc. Jpn. 11, 930 (1956).

[26] M. Reissner, W. Steiner, and H. Boller, ${ }^{57}$ Fe Mössbauer studies on $\mathrm{BaFe}_{2} X_{3}$ compounds with $X=\mathrm{S}$, Se, Hyp. Int. (C) 5, 197 (2002).

[27] K. Komędera, A. K. Jasek, A. Błachowski, K. Ruebenbauer, M. Piskorz, J. Żukrowski, A. Krztoń-Maziopa, E. Pomjakushina, and K. Conder, Magnetism of $\mathrm{BaFe}_{2} \mathrm{Se}_{3}$ studied by Mössbauer spectroscopy, Solid State Commun. 207, 5 (2015).

[28] A. G. Kiiamov, L. R. Tagirov, F. G. Vagizov, D. A. Tayusrkii, H.-A. Krug von Nidda, D. Croitori, V. Tsurkahn, and A. Loidl, Evidence of the plaquette structure of $\mathrm{Fe}_{1+x} \mathrm{Te}$ iron telluride: Mössbauer spectroscopy study, Phys. Status Solidi B 256, 1800698 (2019).

[29] S. E. Dann, M. T. Weller, D. B. Currie, M. F. Thomas, and A. D. Al Rawwas, Structure and magnetic properties of $\mathrm{Sr}_{2} \mathrm{FeO}_{4}$ and $\mathrm{Sr}_{3} \mathrm{Fe}_{2} \mathrm{O}_{7}$ studied by powder neutron diffraction and Mössbauer spectroscopy, J. Mater. Chem. 3, 1231 (1993).

[30] A. V. Sobolev, I. A. Presniakov, A. A. Gippius, I. V. Chernyavskii, M. Schaedler, N. Buettgen, S. A. Ibragimov, I. V. Mozorov, and A. V. Shevelkov, Helical magnetic structure and hyperfine interactions in FeP studied by ${ }^{57} \mathrm{Fe}$ Mössbauer spectroscopy and ${ }^{31} \mathrm{P}$ NMR, J. Alloys. Compd. 675, 277 (2016).

[31] E. Dagotto, Colloquium: The unexpected properties of alkali metal iron selenide superconductors, Rev. Mod. Phys. 85, 849 (2013).

[32] M. Mourigal, S. Wu, M. B. Stone, J. R. Neilson, J. M. Caron, T. M. McQueen, and C. L. Broholm, Block Magnetic Excitations in the Orbitally Selective Mott Insulator $\mathrm{BaFe}_{2} \mathrm{Se}_{3}$, Phys. Rev. Lett. 115, 047401 (2015).

[33] J. Herbrych, J. Heverhagen, N. D. Patel, G. Alvarez, M. Daghofer, A. Moreo, and E. Dagotto, Novel Magnetic Block States in Low-Dimensional Iron-Based Superconductors, Phys. Rev. Lett. 123, 027203 (2019).

[34] N. D. Patel, A. Nocera, G. Alvarez, A. Moreo, S. Johnston, and E. Dagotto, Fingerprints of an orbital-selective Mott phase in the block magnetic state of $\mathrm{BaFe}_{2} \mathrm{Se}_{3}$ ladders, Commun. Phys. 2, 64 (2019).

[35] S. Friedemann, H. Chang, M. B. Gamża, P. Reiss, X. Chen, P. Alireza, W. A. Coniglio, D. Graf, S. Tozer, and F. M. Grosche, Large Fermi surface of heavy electrons at the border of Mott insulating state in $\mathrm{NiS}_{2}$, Sci. Rep. 6, 25335 (2016). 